\title{
The effect of mechanical grinding on the mesoporosity of steam-activated palm kernel shell activated carbons
}

\begin{abstract}
Background: Palm kernel shell activated carbon (OPSA) produced by steam gasification at high temperatures generally results in high surface areas of 1146 to $1600 \mathrm{~m} 2 \mathrm{~g}-1$, attributed to the high volume of micropores $(0.43$ to $0.56 \mathrm{~cm} 3 \mathrm{~g}-1)$. The mesoporosity of naturally occurring activated carbons is observed to increase with decreasing particle size. Mechanical grinding was therefore performed to investigate its effect on the mesoporosity and microporosity of OPSA. Results: Mechanical grinding had a strong effect on mesopore volume and average pore diameter, with an increase in mesopore volume from 47 to $66 \%$ as particle size decreases. Interestingly, no significant effect on the micropore fraction was observed in ground OPSA particles. Conclusions: The mechanically ground OPSA particles possessed dual adsorption capabilities due to the high microporosity and moderate mesoporosity contained in the structures. This results in interesting porosity behaviour of palm kernel shell activated carbons and the potential to provide materials of distinct sorption capacities with minimal treatment.
\end{abstract}

Keyword: Mesoporosity; Microporosity; Palm kernel shell activated carbon; Adsorption isotherms; Mechanical grinding 